\title{
Low Threshold, Wide Dynamic Range, Tunable, All-Optical Self-Modulator Based on Fano Resonance and Out-of-Plane Coupling in a Slab Photonic Crystal with a Graphene Layer
}

\author{
Reza Asadi and Zhengbiao Ouyang \\ THz Technical Research Center, Shenzhen Key Laboratory of Micro-nano Photonic Information Technology, \\ College of Electronic Science and Technology, Shenzhen University, Shenzhen 518060, China \\ Correspondence should be addressed to Zhengbiao Ouyang; zbouyang@szu.edu.cn
}

Received 3 August 2015; Revised 31 October 2015; Accepted 12 November 2015

Academic Editor: Jorge M. Seminario

Copyright (c) 2015 R. Asadi and Z. Ouyang. This is an open access article distributed under the Creative Commons Attribution License, which permits unrestricted use, distribution, and reproduction in any medium, provided the original work is properly cited.

We demonstrate an all-optical modulator based on self-modulation in a one-dimensional slab photonic crystal (PhC) by using optical Kerr nonlinearity of graphene and Fano resonance effect. It has been shown that the effect of Fano resonance in a onedimensional slab $\mathrm{PhC}$ for intensity enhancement can provide low threshold $\left(\sim 1 \mathrm{MW} / \mathrm{cm}^{2}\right)$, high frequency $(>1 \mathrm{THz})$, and wide dynamic range $(>3 \mathrm{THz})$ tunability for the all-optical self-modulator. Such a self-modulator can find applications in optical pulse generations, optical clocks, frequency shifting, and so forth.

\section{Introduction}

In recent years, research on $\mathrm{THz}$ sources for applying in $\mathrm{THz}$ science and technology is attracting great attention. Up to now various optical and electrical methods have been used for $\mathrm{THz}$ sources [1-11]; however at present, achieving compact, efficient, and economic sources remains a challenge to scientists and engineers [11-14]. One of the main optical methods for CW THz wave generation is heterodyne method that uses $\mathrm{THz}$ beat frequency of two frequency-locked laser beams with two different frequencies $[10,11]$. In heterodyne method, the $\mathrm{THz}$ beat frequency can be tuned by changing the optical frequency of lasers. However this tuning is generally not so easy and requires a complex optical system. Also, other technical difficulties and high cost due to using two different lasers and problems due to optical alignments and frequency locking lead to the fact that this method is mainly used only in laboratories to characterize the $\mathrm{THz}$ antenna and devices.

To overcome the mentioned difficulties and complexity, we already proposed a compact size and easy tunable optical modulator based on all-optical self-modulation in a slab photonic crystal $(\mathrm{PhC})$ waveguide with a single graphene layer under the condition of in-plane illumination from the waveguide. In this structure, by using the ability of optical resonance at higher photonic bandgap edges with relatively high quality factor, we found all-optical sub-THz $(\sim$ $0.6 \mathrm{THz}$ ) self-modulation with relatively low input intensity $\left(\sim 15 \mathrm{MW} / \mathrm{cm}^{2}\right)$. We have also shown that the $\mathrm{THz}$ modulation frequency can be tuned easily by tuning input intensity with tuning sensitivity (tunability versus input intensity) of $0.04 \mathrm{THz} /\left(\mathrm{MW} / \mathrm{cm}^{2}\right)$. In that structure, however, a limitation is that one can have regular oscillation only in a small dynamic range around the threshold intensity so that the dynamic range of modulation frequency is relatively small (in the order of $0.1 \mathrm{THz}$ ). In addition, we have shown that, in that structure to achieve higher modulation frequencies $(\sim 3 \mathrm{THz})$, one have to use lower photonic bands with lower quality factors; however this leads to requiring much higher threshold intensities more than $100 \mathrm{MW} / \mathrm{cm}^{2}$.

To overcome the mentioned limitations, here we propose using out-of-plane coupling instead of in-plane coupling. In the case of out-of-plane coupling we use the ability of Fano resonance effect (instead of optical resonance at photonic bandgap edges) for achieving light intensity enhancement inside the PhC. 
Considering page limit, the self-modulator is aimed for application in $\mathrm{THz}$ wave generation in this paper. However, the principle is the same for other applications in $\mathrm{THz}$ band and various applications in other frequency bands. We will show that Fano resonances of higher photonic bands can provide suitable quality factors to obtain relatively lower threshold intensity (in comparison with in-plane coupling) for achieving self-modulation, especially for modulation frequencies higher than $1 \mathrm{THz}$. In addition, we will show that instead of in-plane coupling, using out-of-plane coupling and Fano resonance effect provides larger dynamic range of modulation frequency more than $3 \mathrm{THz}$.

Furthermore, choosing out-of-plane illumination leads to much easier implementation without requiring optical alignment (compared with coupling inside the $\mathrm{PhC}$ waveguide). Thus the proposed $\mathrm{THz}$ self-modulator is expected to have relatively wide applications in $\mathrm{THz}$ science and technology due to its compactness, easy implementation, and wide dynamic range of frequency tuning.

\section{Physical Model of the THz Self-Modulator Proposed}

The schematic of the proposed structure is shown in Figure 1. The structure includes a $\mathrm{SiO}_{2}$ substrate and a $1 \mathrm{D}$ silicon $(\mathrm{Si})$ $\mathrm{PhC}$ with 9 periods and a single layer of graphene in the middle cell of the PhC. The filling factor $(F=w / a)$ of $\mathrm{Si}$ in the $\mathrm{PhC}$ is 0.9 and the thickness of Si layer is $h=0.5 a$, where $a$ is the period of the $\mathrm{PhC}$ that is selected by considering that the operation wavelength corresponds to the communication wavelength of $\lambda=1550 \mathrm{~nm}$ of the input laser wave and $w$ is the width of each Si layer as shown in Figure 1. As we will show in the next sections, for our purpose the suitable normalized frequency $(a / \lambda)$ is about 0.966 , so we will have $a \approx 1497 \mathrm{~nm}$. The horizontal width of the graphene layer is the same as that of the silicon layer in the PhC unit cell. For the illumination, we consider a light polarized in $z$-direction inputted from the upside, as shown in Figure 1.

For fabrication of this structure, two extra processes are needed in comparison with usual Si PhC, that is, bonding and etching of graphene. Until now, various techniques have been developed for these processes which are also in rapid progress. For example, for graphene bonding one can use annealing $[15,16]$ or anodic bonding [17] and for graphene etching one can use chemical etching [18]. It is noticeable that, in the annealing bonding method, the interaction between graphene and substrate is due to the Van der Waals force that leads to a small band gap between valance and conductive bands of the graphene in the order of few $0.1 \mathrm{eV}$ [19], so, as Gao et al. [19] show, this small perturbation has negligible effect on other parts of electronic band structure. Therefore, considering that our desired optical frequency is much higher than the induced band gap, the change in linear and nonlinear optical properties of graphene caused from graphene bonding can be neglected. This result also can be found from the fact that experimental results for nonlinear optical properties of multilayer graphene (that are bonded by Van der Waals force) in optical frequencies are the same as that of single layer of graphene [20].

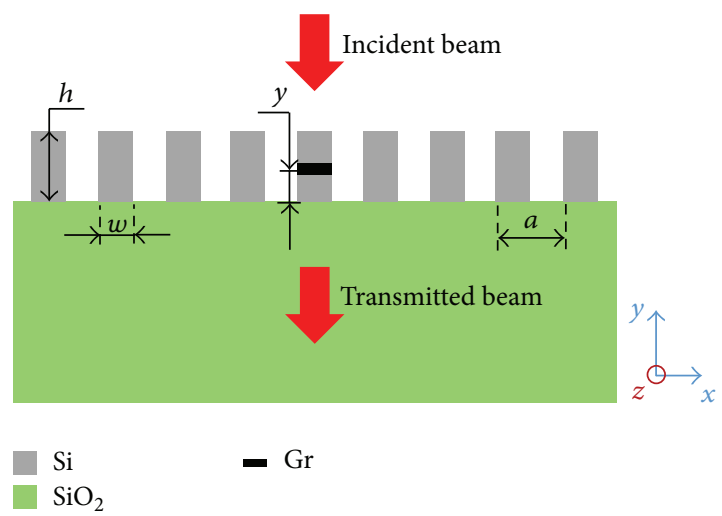

FIgURE 1: Schematic of the $\mathrm{THz}$ modulator. It consists of a onedimensional (1D) slab $\mathrm{PhC}$ and a layer of graphene (Gr). A continuous infrared wave with a wavelength of $1550 \mathrm{~nm}$ is inputted from upside and self-modulated after transmission from the structure.

\section{Theory and Calculation Method}

In order to determine the suitable normalized frequency, we first calculate the transmission spectrum (versus normalized frequency) for the out-of-plane illumination with incident angle of zero. For calculation of transmission coefficient, we use an incident beam with a Gaussian profile with full width at half maximum (FWHM) of $5 a$. This size has been selected to be comparable with the size of usual optical fibers. We calculate the transmission and then we perform nonlinear FDTD simulation to calculate the change of $\mathrm{PhC}$ transmission for different intensities of the pump beam at the selected normalized frequency.

We consider the dielectric constants of $\mathrm{Si}$ and $\mathrm{SiO}_{2}$ as 12 and 2.1, respectively. Noting that the thickness of the graphene $\left(d_{g}=0.34 \mathrm{~nm}\right)$ is much smaller than the wavelength, thus we can consider the graphene and silicon around it (with a thickness equal to one mesh size of $0.02 a \ll \lambda$ ) as a composite layer with an equivalent dielectric constant $[21,22]$ as

$$
\varepsilon_{c}=\varepsilon_{d}+i \frac{\sigma_{0}}{\omega \varepsilon_{0} d_{g}} \rho,
$$

where $\varepsilon_{d}$ is the dielectric constant of $\mathrm{Si}, \sigma_{0}=6.08 \times 10^{-5} \Omega$ is the initial conductivity of the graphene, and $\rho$ is the filling factor of the graphene inside the composite layer. $\omega$ is the angular frequency of light wave and $\varepsilon_{0}$ is the vacuum permittivity. The increment of the equivalent dielectric constant of the composite layer under the illumination of an input wave with intensity $(I)$ is calculated by using the following equations:

$$
\begin{gathered}
\Delta \varepsilon_{c}=\rho \Delta \varepsilon_{g}, \\
\Delta \varepsilon_{g}=\Delta\left(n_{g}^{2}\right), \\
\frac{d}{d t} \Delta n_{g}=\frac{\beta n_{2} I}{\tau_{c}}-\frac{\Delta n_{g}}{\tau_{c}},
\end{gathered}
$$

where $\varepsilon_{g}$ and $n_{g}$ are the dielectric constant and refractive index of graphene, respectively, $\tau_{c}=100 \mathrm{fs}$ [23] is the fall 


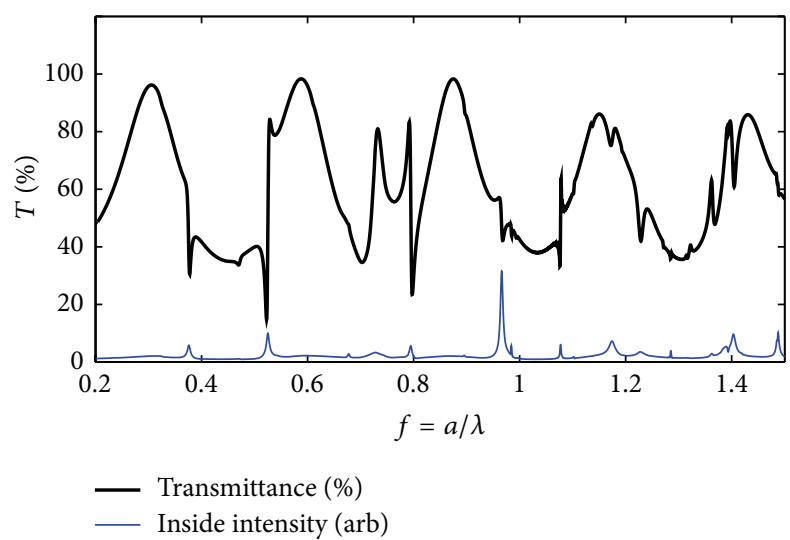

FIgURE 2: The transmission coefficient $(T)$ and intensity inside the $\mathrm{PhC}$ (at the middle of the $\mathrm{PhC}$ ) versus normalized frequency $(f=$ $a / \lambda)$ for the case of $\mathrm{PhC}$ without graphene.

time of the refractive index change, $\beta=1 /\left(1+I / I_{s}\right), n_{2}=$ $10^{-7} \mathrm{~cm}^{2} / \mathrm{W}$, and $I_{s}=600 \mathrm{MW} / \mathrm{cm}^{2}[20]$.

As in our precious works [24], considering that there exists negative absorption nonlinearity of graphene that leads to reduction of its absorption with increasing light intensity, we neglect the effect of absorption nonlinearity and only consider Kerr nonlinearity of the graphene.

\section{Results and Discussions}

In Figure 2, the calculation result for the transmission coefficient of the PhC without graphene for normal incident angle (bold line) and the intensity at the position of the middle of the $\mathrm{PhC}$ (thin line) is shown. As can be seen, at the special normalized frequencies $(f)$, some Fano resonances take place, leading to relatively sharp change in $\mathrm{PhC}$ transmittance. Also as can be seen the maximum intensity inside the $\mathrm{PhC}$ and equivalently the maximum quality factor is obtained for the Fano resonance at $f=0.966$. The advantage of using Fano resonance with higher quality factor is its higher sensitivity to the change of the refractive index of the $\mathrm{PhC}$ in addition to providing higher intensity enhancement. These two factors help to decrease the required input intensity (threshold intensity) to achieve nonlinear effects.

The result of nonlinear FDTD simulations, for the PhC output power versus time for a number of fixed incident light intensities for normalized frequency of 0.966 (corresponding to the strongest Fano resonance), has been plotted in Figure 3.

As can be seen from Figure 3, for the intensities higher than $1.5 \mathrm{MW} / \mathrm{cm}^{2}$ (we name it threshold intensity) selfmodulation takes place. The regular oscillation takes place up to intensity of $\sim 10 \mathrm{MW} / \mathrm{cm}^{2}$ and for much higher intensities (Figure 3(b)) the oscillation regularity decreases. Also by increasing the input intensity the modulation frequency increases. This ability provides an easy method for tuning the modulation frequency without requiring tuning optical frequency as it is needed in $\mathrm{THz}$ sources based on heterodyne method.
To study the modulation frequency tunability in more detail, in Figure 4 (red line), the modulation frequency $\left(F_{M}\right)$ versus input intensity for the strongest Fano resonance $(f=$ $0.966)$ is shown. In addition, for comparison, the result of selfmodulation for the other Fano resonance at lower normalized frequency of $f=0.527$ has been shown by blue line in Figure 4. As can be seen, for the case of strongest Fano resonance $(f=0.966)$ for intensities from 1.5 to $10 \mathrm{MW} / \mathrm{cm}^{2}$, the modulation frequency increases from about $1 \mathrm{THz}$ to $\sim$ $4 \mathrm{THz}$. That corresponds to tuning sensitivity of $d F_{M} / d I \approx$ $0.35 \mathrm{THz} /\left(\mathrm{MW} / \mathrm{cm}^{2}\right)$.

Thus, in comparison with our previous work [19], the dynamic range of modulation frequency tunability increases more than one order of magnitude $\left(\Delta F_{M}>3 \mathrm{THz}\right)$ and the tuning sensitivity $\left(d F_{M} / d I\right)$ also increases about one order of magnitude in addition to decreasing the required threshold intensity.

Also, as can be seen from Figure 4, for Fano resonance at $f=0.527$, the dynamic range of tunability of $\mathrm{THz}$ modulation frequency is approximately the same as for $f=$ 0.966 , but the required input intensity is increased strongly specially at higher modulation frequencies.

In order to study the effect of more graphene layers instead of one graphene layer in Figure 1, the results of the calculation of self-modulation frequency at normalized frequency of $f_{0}=0.966$ for various number of graphene layers $(N)$ have been shown in Figure 5 . As can be seen, for $N=2$ and $N=3$, the dynamic range of modulation frequency increases a little in comparison with $N=1$ and the maximum regular modulation frequency is about $5 \mathrm{THz}$. Also, the threshold intensity for $N=2$ and $N=3$ decreases to less than $1 \mathrm{MW} / \mathrm{cm}^{2}$ and the tuning sensitivity $\left(d F_{M} / d I\right)$ increases up to $\sim 0.9 \mathrm{THz} /\left(\mathrm{MW} / \mathrm{cm}^{2}\right)$ and $\sim 1.3 \mathrm{THz} /\left(\mathrm{MW} / \mathrm{cm}^{2}\right)$, respectively. Thus, by using only 3 graphene layers we can obtain modulation frequency higher than $3 \mathrm{THz}$ with input intensities less than $3 \mathrm{MW} / \mathrm{cm}^{2}$.

For further study, the effect of more unit cells that contain a graphene layer (as shown in Figures 6(a) and 6(b)) on the modulation frequency and sensitivity has been shown in Figure 6(c). As can be seen, with the increase of the unit cells that contain a graphene layer $\left(N_{d}\right)$, the dynamic range of modulation frequency and sensitivity (the slope of the curve $F_{M}$ versus $I$ ) decreases and the threshold intensity increases.

The mechanism of the self-modulation can be described as follows. Consider the resonance condition that the optical frequency of the incident beam corresponds to the Fano resonance frequency. Thus the $\mathrm{PhC}$ behaves as an optical cavity. At first, the field in the cavity is 0 or very weak; no nonlinear effect exists; after a time period of building up field in the cavity due to nonzero quality factor of the cavity (or nonzero response time of the $\mathrm{PhC}$ ), the field in the cavity reaches a high value and then nonlinear effects take place and refractive index of the cavity changes, resulting in detuning of the cavity (or Fano resonance frequency) that leads to decreasing the field in the cavity in comparison with the resonance condition (equality of Fano resonance frequency and light frequency); then after some time, the field in the cavity decreases to be very weak so that 


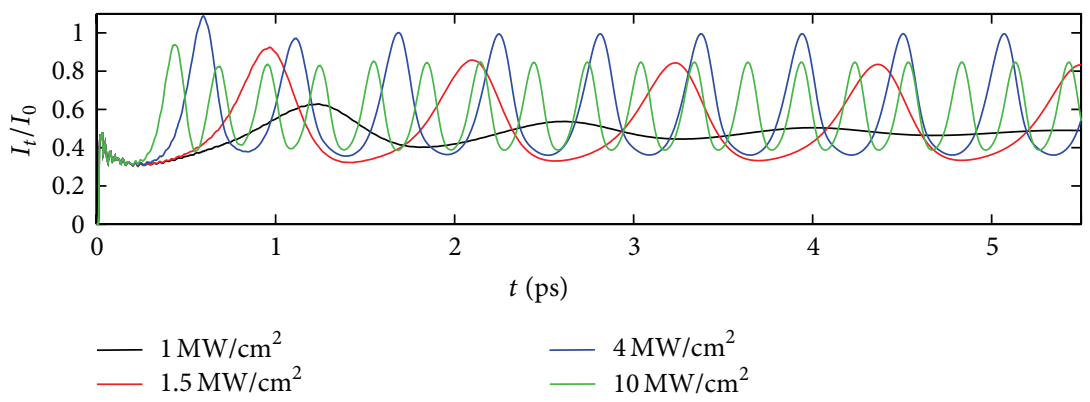

(a)

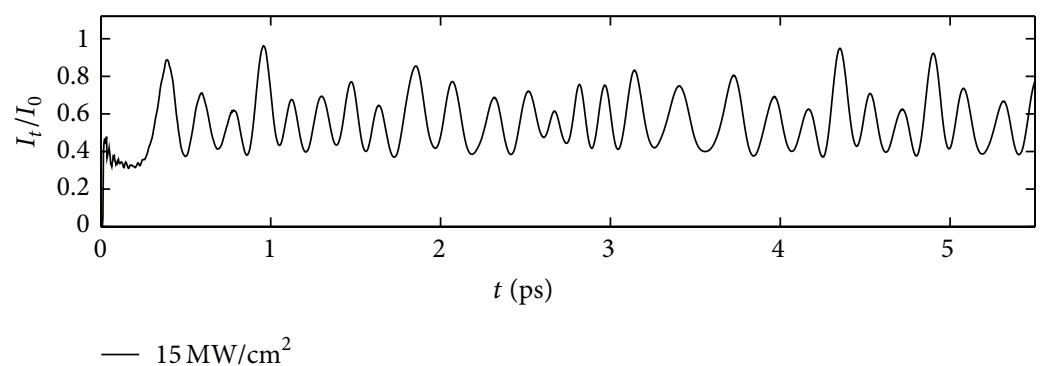

(b)

FIGURE 3: The transmission power versus time for a number of fixed average intensities of the incidence beam at the normalized frequency of $f_{0}=0.966$ (corresponding to the peak of the strongest Fano resonance). $I_{t}$ is the transmitted intensity and $I_{0}$ is the incident intensity. The inset numbers show the incident intensities.

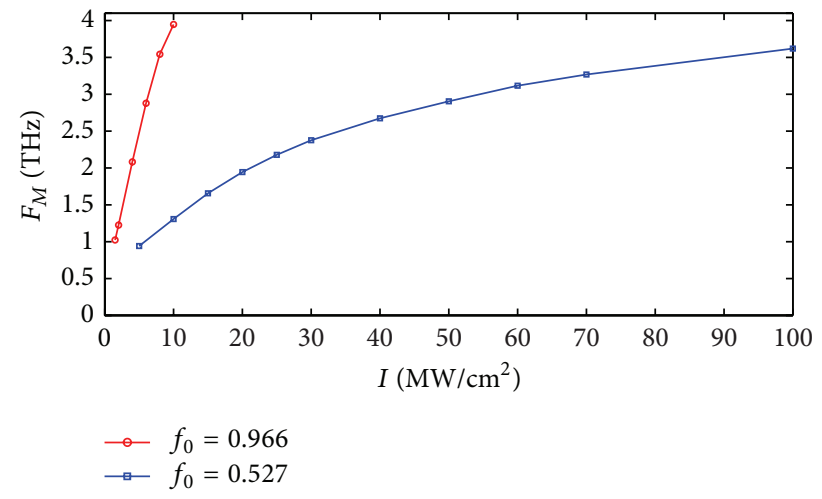

FIGURE 4: The modulation frequency $\left(F_{M}\right)$ versus average intensity of the incidence beam for normalized frequency of $f_{0}=0.966$ (red line) and $f_{0}=0.527$ (blue line).

the refractive index of the cavity returns or approaches to its initial value that leads the cavity to return to its initial resonance condition. Obviously, such a process would repeat in the system. Such a repeated process accounts for the selfmodulation phenomenon in the proposed structure.

Also the contrary effect of the increasing of the graphene layers in one unit cell (Figure 5) and in multiunit cells (Figure 6) can be explained as follows. The increasing of the unit cells that contain a graphene layer (case A) leads to increasing of absorption of the $\mathrm{PhC}$ that leads to decreasing of the Fano resonance quality factor and thus decreasing of the sensitivity of the Fano resonance shift with the change of refractive index. But in the case of increasing the number of the graphene layers only in one unit cell (case B), although

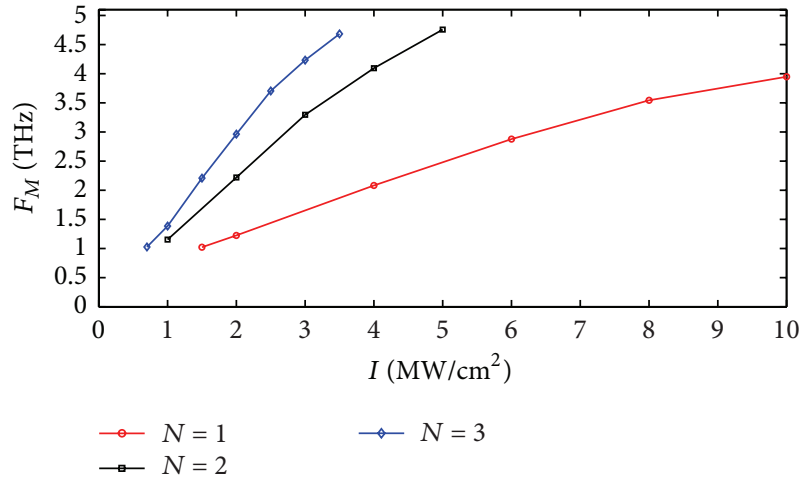

FIGURE 5: The modulation frequency $\left(F_{M}\right)$ versus average intensity of the incidence beam for normalized frequency of $f_{0}=0.966$ (peak of the strongest Fano resonance in Figure 2) for various number $(N)$ of graphene layers in the center cell in Figure 1.

the absorption increases (like case A), the average change of refractive index of the $\mathrm{PhC}$ is more than that in case $\mathrm{A}$, because the intensity inside the middle cell is higher and also increases more due to the self-focusing effect. These two factors help to improve the sensitivity of the shift of the Fano resonance and thus the sensitivity of the modulation frequency

\section{Conclusions}

It has been shown that using Fano resonance effect and out-of-plane illumination in $1 \mathrm{D}$ slab PhCs that consist of a single layer of graphene can provide wide dynamic range 


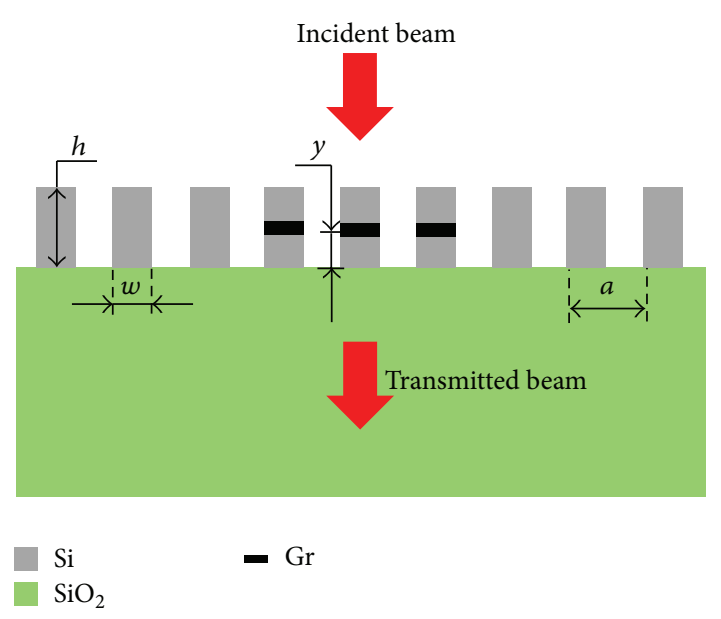

(a)

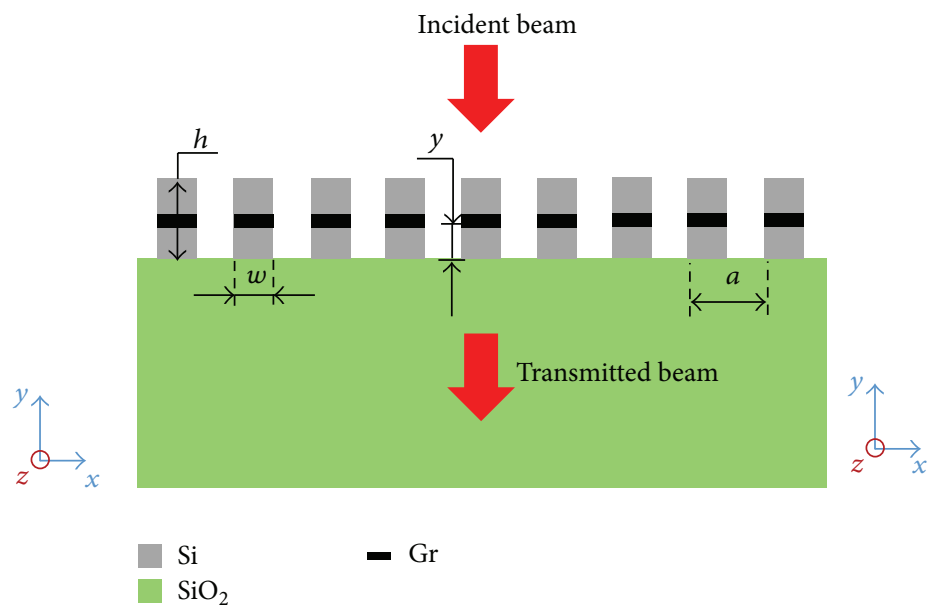

(b)

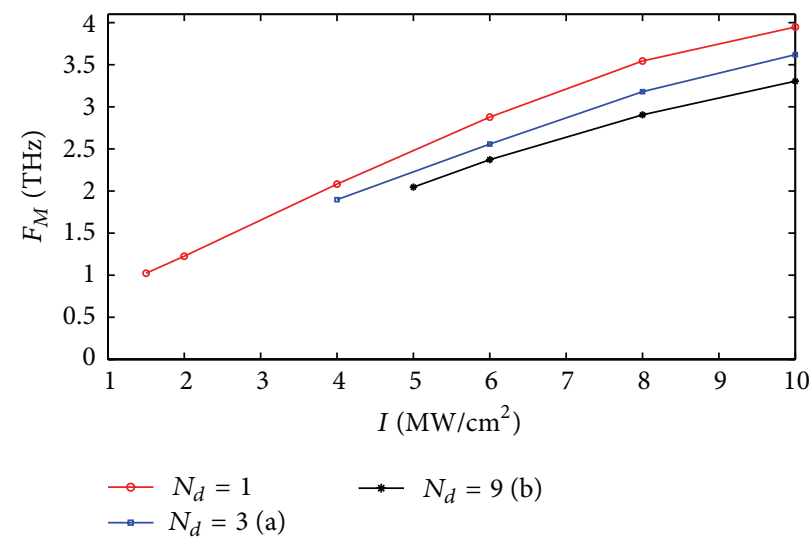

(c)

FIGURE 6: Self-modulators with 1, 3, and 9 silicon layers each embedded with a single graphene layer.

of tunability ( $>3 \mathrm{THz}$ ) from about $1 \mathrm{THz}$ to $\sim 4 \mathrm{THz}$ selfmodulation frequency with only tuning of input intensity (without requiring change of optical frequency). Also we have shown that using only 3 graphene layers can decrease the required input intensity less than $3 \mathrm{MW} / \mathrm{cm}^{2}$ for modulation frequencies up to $4 \mathrm{THz}$.

Thus in comparison with our previous work that we used resonance effect at photonic band gap edges [19], using Fano resonance effect can improve the performance of selfmodulation in nonlinear slab $\mathrm{PhC}$ in parameters including dynamic range of tunability, tuning sensitivity $\left(d F_{M} / d I\right)$, and threshold intensity. In addition, in comparison with inplane illumination, out-of-plane illumination can be used with easier $\mathrm{PhC}$ structure without requiring waveguide (for light coupling) and also easier implementation without requiring high-accuracy optical coupling and alignment. So, the proposed self-modulator can be expected to have wide applications in $\mathrm{THz}$ systems.

\section{Conflict of Interests}

The authors declare that there is no conflict of interests regarding the publication of this paper.

\section{Acknowledgments}

This work was supported by the NSFC (Grant nos. 61275043, 61171006, 61107049, and 60877034), the Guangdong Province NSF (Key Project, Grant no. 8251806001000004), and the Shenzhen Science Bureau (Grant nos. 200805, CXB201105050064A).

\section{References}

[1] P. Zhao, S. Ragam, Y. J. Ding, and I. B. Zotova, “Terahertz intracavity generation from output coupler consisting of stacked GaP plates," Applied Physics Letters, vol. 101, no. 2, Article ID 021107, 2012.

[2] T. J. Edwards, D. Walsh, M. B. Spurr, C. F. Rae, M. H. Dunn, and P. G. Browne, "Compact source of continuously and widelytunable terahertz radiation,” Optics Express, vol. 14, no. 4, pp. 1582-1589, 2006.

[3] F. Kadlec, P. Kuzel, and J. Coutaz, "Study of terahertz radiation generated by optical rectification on thin gold films," Optics Letters, vol. 30, no. 11, p. 1402, 2005.

[4] R. Köhler, A. Tredicucci, F. Beltram et al., "Terahertz semiconductor-heterostructure laser," Nature, vol. 417, no. 6885, pp. 156-159, 2002. 
[5] S. Kumar, B. S. Williams, S. Kohen, Q. Hu, and J. L. Reno, "Continuous-wave operation of terahertz quantum-cascade lasers above liquid-nitrogen temperature," Applied Physics Letters, vol. 84, no. 14, pp. 2494-2496, 2004.

[6] D. G. Paveliev, Y. I. Koshurinov, A. S. Ivanov et al., "Experimental study of frequency multipliers based on a GaAs/AlAs semiconductor superlattices in the terahertz frequency range," Semiconductors, vol. 46, no. 1, pp. 121-125, 2012.

[7] A. Maestrini, J. S. Ward, J. J. Gill et al., "A frequency-multiplied source with more than $1 \mathrm{~mW}$ of power across the $840-900-\mathrm{GHz}$ band," IEEE Transactions on Microwave Theory and Techniques, vol. 58, no. 7, pp. 1925-1932, 2010.

[8] L. Li, L. A. Yang, J. C. Zhang et al., "Threading dislocation reduction in transit region of GaN terahertz Gunn diodes," Applied Physics Letters, vol. 100, no. 7, Article ID 072104, 2012.

[9] B. A. Knyazev, G. N. Kulipanov, and N. A. Vinokurov, "Novosibirsk terahertz free electron laser: instrumentation development and experimental achievements," Measurement Science and Technology, vol. 21, Article ID 054017, 2010.

[10] S. Preu, G. H. Döhler, S. Malzer, L. J. Wang, and A. C. Gossard, "Tunable, continuous-wave Terahertz photomixer sources and applications," Journal of Applied Physics, vol. 109, no. 6, Article ID 061301, 2011.

[11] E. Rouvalis, C. C. Renaud, D. G. Moodie, M. J. Robertson, and A. J. Seeds, "Traveling-wave uni-traveling carrier photodiodes for continuous wave THz generation," Optics Express, vol. 18, no. 11, pp. 11105-11110, 2010.

[12] B. Ferguson and X.-C. Zhang, "Materials for terahertz science and technology," Nature Materials, vol. 1, no. 1, pp. 26-33, 2002.

[13] M. Tonouchi, "Cutting-edge terahertz technology," Nature Photonics, vol. 1, no. 2, pp. 97-105, 2007.

[14] A. Maestrini, B. Thomas, H. Wang et al., "Schottky diode-based terahertz frequency multipliers and mixers," Comptes Rendus Physique, vol. 11, no. 7-8, pp. 480-495, 2010.

[15] T. Ikuta, K. Gumi, Y. Ohno, K. Maehashi, K. Inoue, and K. Matsumoto, "Direct graphene synthesis on a $\mathrm{Si} / \mathrm{SiO}_{2}$ substrate by a simple annealing process," Materials Research Express, vol. 1, no. 2, Article ID 025028, 2014.

[16] Z. Wang and E. Liu, "Graphene ultrathin film electrode for detection of lead ions in acetate buffer solution," Talanta, vol. 103, pp. 47-55, 2013.

[17] W. Jung, T. Yoon, J. Choi et al., "Superstrong encapsulated monolayer graphene by the modified anodic bonding," Nanoscale, vol. 6, no. 1, pp. 547-554, 2014.

[18] X. Wang and H. Dai, "Etching and narrowing of graphene from the edges," Nature Chemistry, vol. 2, no. 8, pp. 661-665, 2010.

[19] W. Gao, P. Xiao, G. Henkelman, K. M. Liechti, and R. Huang, "Interfacial adhesion between graphene and silicon dioxide by density functional theory with van der Waals corrections," Journal of Physics D: Applied Physics, vol. 47, no. 25, Article ID 255301, 2014.

[20] H. Zhang, S. Virally, Q. Bao et al., "Z-scan measurement of the nonlinear refractive index of graphene," Optics Letters, vol. 37, no. 11, pp. 1856-1858, 2012.

[21] L. A. Falkovsky, "Optical properties of graphene," Journal of Physics: Conference Series, vol. 129, Article ID 012004, 2008.

[22] M. A. K. Othman, C. Guclu, and F. Capolino, "Graphenedielectric composite metamaterials: evolution from elliptic to hyperbolic wavevector dispersion and the transverse epsilonnear-zero condition," Journal of Nanophotonics, vol. 7, Article ID 073089, 2013.
[23] M. Breusing, C. Ropers, and T. Elsaesser, "Ultrafast carrier dynamics in graphite," Physical Review Letters, vol. 102, no. 8, Article ID 086809, 2009.

[24] R. Asadi, Z. Ouyang, and M. M. Mohammd, "A compact, alloptical, $\mathrm{THz}$ wave generator based on self-modulation in a slab photonic crystal waveguide with a single sub-nanometer graphene layer," Nanoscale, vol. 7, no. 26, pp. 11379-11385, 2015. 

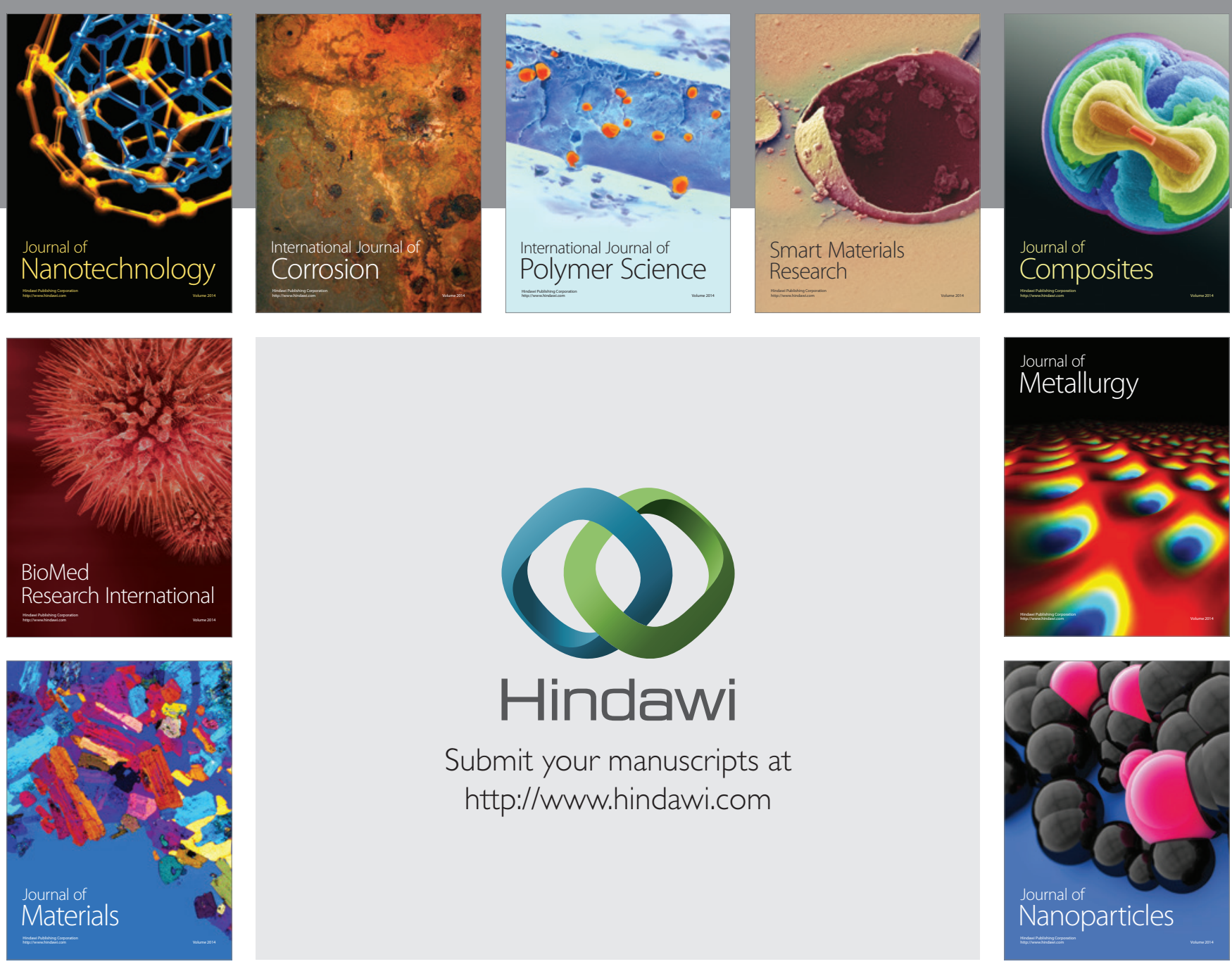

Submit your manuscripts at http://www.hindawi.com
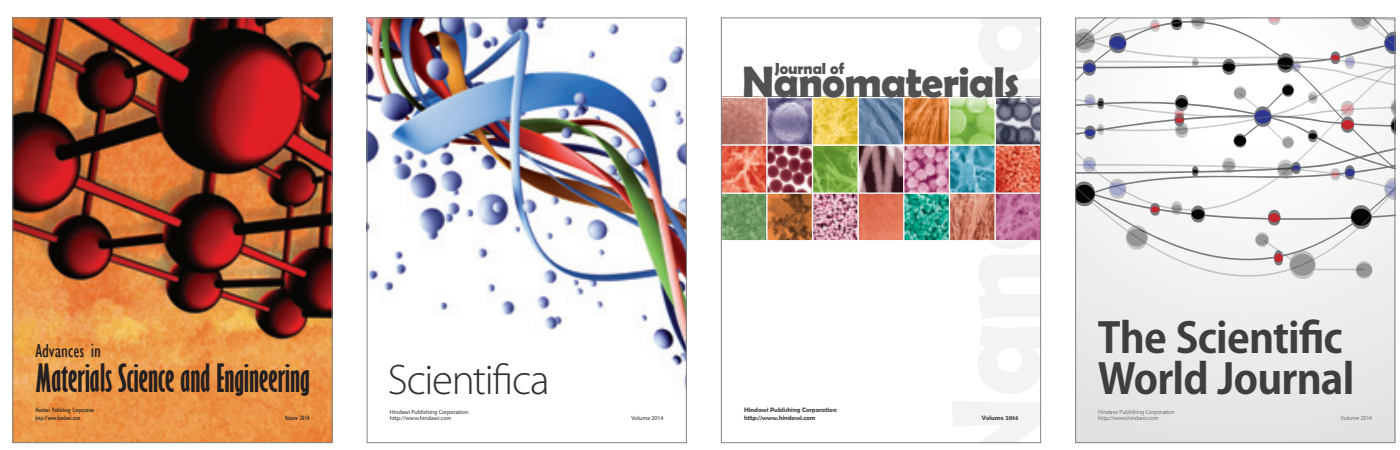

\section{The Scientific World Journal}
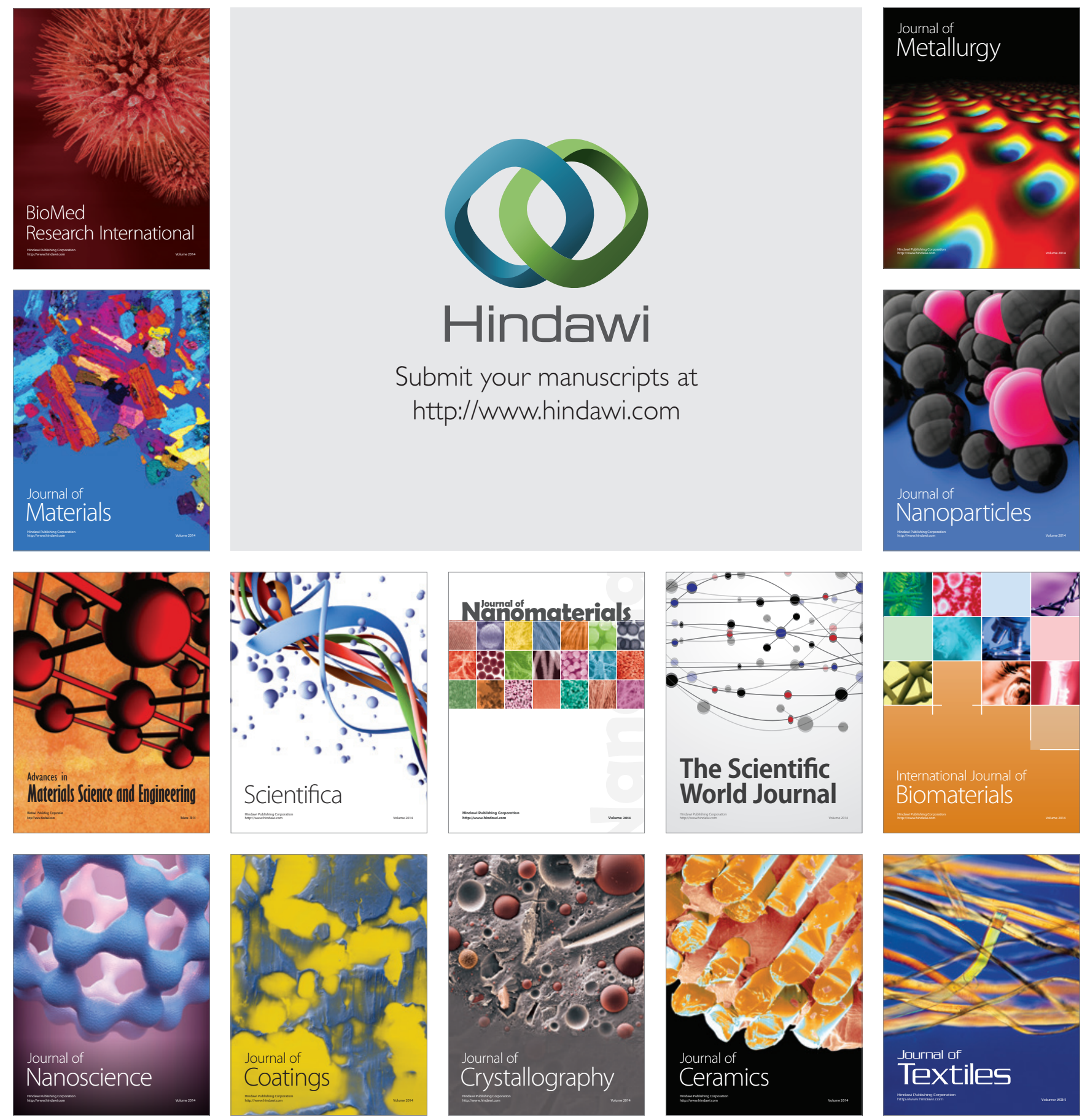